\title{
CBV_ASPECTS Improvement over CT_ASPECTS on Determining Irreversible Ischemic Lesion Decreases over Time
}

\author{
Marina Padroni ${ }^{a} \quad$ Sandra Boned $^{b}$ Marc Ribó $^{b} \quad$ Marian Muchada ${ }^{b}$ \\ David Rodriguez-Luna ${ }^{b}$ Pilar Coscojuelac Alejandro Tomasello ${ }^{c}$ Jordi Cabero ${ }^{c}$ \\ Jorge Pagolab Noelia Rodriguez-Villatoro $^{b}$ Jesus M. Juega ${ }^{b}$ Estela Sanjuan ${ }^{b}$ \\ Carlos A. Molina ${ }^{b}$ Marta Rubierab \\ a Section of Neurology, Department of Biological, Psychiatric and Psychological Science, University \\ of Ferrara, Ferrara, Italy; ${ }^{b}$ Stroke Unit, Hospital Universitari Vall d'Hebron, Universidad Autónoma de \\ Barcelona, and 'Neuroradiology Unit, Hospital Universitari Vall D'Hebron, Barcelona, Spain
}

\section{Key Words}

Computed tomography $\cdot$ Computed tomography perfusion $\cdot$ Stroke $\cdot$ Thrombectomy

\begin{abstract}
The Alberta Stroke Program Early CT Score (ASPECTS) is a useful scoring system for assessing early ischemic signs on noncontrast computed tomography (CT). Cerebral blood volume (CBV) on $\mathrm{CT}$ perfusion defines the core lesion assumed to be irreversibly damaged. We aim to explore the advantages of CBV_ASPECTS over CT_ASPECTS in the prediction of final infarct volume according to time. Methods: Consecutive patients with anterior circulation stroke who underwent endovascular reperfusion according to initial CT_ASPECTS $\geq 7$ were studied. CBV_ASPECTS was assessed blindly later on. Recanalization was defined as thrombolysis in cerebral ischemia score $2 b-3$. Final infarct volumes were measured on follow-up imaging. We compared ASPECTS on CBV and CT images, and defined ASPECTS agreement as: CT_ASPECTS - CBV_ASPECTS $\leq 1$. Results: Sixty-five patients, with a mean age of $67 \pm 14$ years and a median National Institutes of Health Stroke Scale score of 16 (range 10-20), were studied. The recanalization rate was $78.5 \%$. The median CT_ASPECTS was 9 (range $8-10$ ), and the CBV_ ASPECTS was 8 (range 8-10). The mean time from symptoms to CT was $219 \pm 143$ min. Fifty patients (76.9\%) showed ASPECTS agreement. The ASPECTS difference was inversely correlated to the time from symptoms to CT $(r=-0.36, p<0.01)$. A ROC curve defined 120 min as the best cutoff point after which the ASPECTS difference becomes more frequently $\leq 1$. After $120 \mathrm{~min}, 89.5 \%$ of the patients showed ASPECTS agreement (as compared with $37.5 \%$ for $<120$ $\min , p<0.01)$. CBV_ASPECTS but not CT_ASPECTS correlated with final infarct $(r=-0.33, p<$
\end{abstract}

Additional data can be obtained regarding research objectives contacting the corresponding author (mrubifu@ hotmail.com). 
0.01). However, if CT was done $>2 \mathrm{~h}$ after symptom onset, CT_ASPECTS also correlated to final infarct $(r=-0.39, p=0.01$ ). Conclusions: In acute stroke, CBV_ASPECTS correlates with the final infarct volume. However, when CT is performed after 120 min from symptom onset, CBV_ASPECTS does not add relevant information to CT_ASPECTS.

(C) 2016 S. Karger AG, Basel

\section{Introduction}

The optimal imaging paradigm for selecting acute ischemic stroke patients (AIS) for reperfusion therapies is not defined yet. The Alberta Stroke Program Early CT Score (ASPECTS) [1] is a useful scoring system to assess the extent of early ischemic signs in the middle cerebral artery (MCA) territory on noncontrast computed tomography (CT). The ASPECTS has been demonstrated to be associated with outcome in patients receiving intravenous as well as endovascular reperfusion therapies [2-4]. However, there is no complete agreement on considering ASPECTS a good prognostic marker [5, 6]. Its main limitations are the modest interobserver agreement (especially when dichotomized at 7 [7]) and the lack of information on the extent of ischemic penumbra [8]. Furthermore, the meaning of CT early ischemic changes is not unequivocal: only parenchymal hypoattenuation (focal hypodensity and/or loss of gray-white matter differentiation) represents irreversibly injured brain tissue while isolated cortical swelling may suggest penumbral or oligemic tissue [9].

In the last years, multimodal CT [including CT, CT angiography (CTA) and CT perfusion (CTP)] has been extensively used for the selection of AIS patients for endovascular reperfusion treatments in clinical trials. EXTEND-IA [10] demonstrated the effectiveness of mechanical thrombectomy in patients with large-vessel occlusion and salvageable tissue on CTP. In MR RESCUE [11], the subgroup of patients with a 'penumbral pattern' on CTP achieved a good functional outcome regardless of treatment assignment.

Cerebral blood volume (CBV) maps on CTP have been classically used to define the core lesion assumed to be irreversibly damaged $[12,13]$. ASPECTS can be applied to CTP maps to improve the prediction of the final infarct extent and stroke outcome [14]. However, recent evidences suggest that CBV maps could overestimate the final infarct volume [15]. Therefore, the optimal CBV ASPECTS threshold to discriminate between AIS patients with good and poor clinical outcome remains to be established [14, 16-18]. Moreover, whether CBV provides more additional information compared to CT in the initial assessment of AIS patients is still a matter of controversy. We aim to explore the advantages of CBV_ASPECTS over CT_ASPECTS in the prediction of final infarct volume, as a surrogate marker of clinical outcome.

\section{Methods}

From January 2012 to July 2015, we studied consecutive patients with MCA (M1 and proximal M2) or internal carotid artery (ICA) occlusion below $8 \mathrm{~h}$ from symptom onset who received a multimodal CT at baseline and underwent endovascular reperfusion treatment. All patients were selected for endovascular treatment according to our local protocol, including a baseline favorable functional status [modified Rankin scale ( $\mathrm{mRS}$ ) score $<3$ ] and an initial CT_ASPECTS $\geq 7$. Mechanical thrombectomies were performed by experienced interventionalists using commercially available stent retrievers and aspiration catheters. At the end of the procedure, recanalization was determined by the thrombolysis in cerebral ischemia (TICI) score. Complete recanalization was defined as TICI score $2 \mathrm{~b}$ or 3 . Long-term outcome was assessed at 3 months by means of the mRS considering as good outcome an mRS score $<3$.

The study was approved by our local Ethics Committee. Informed consent was obtained from each patient or from his/her close relatives on admission. 

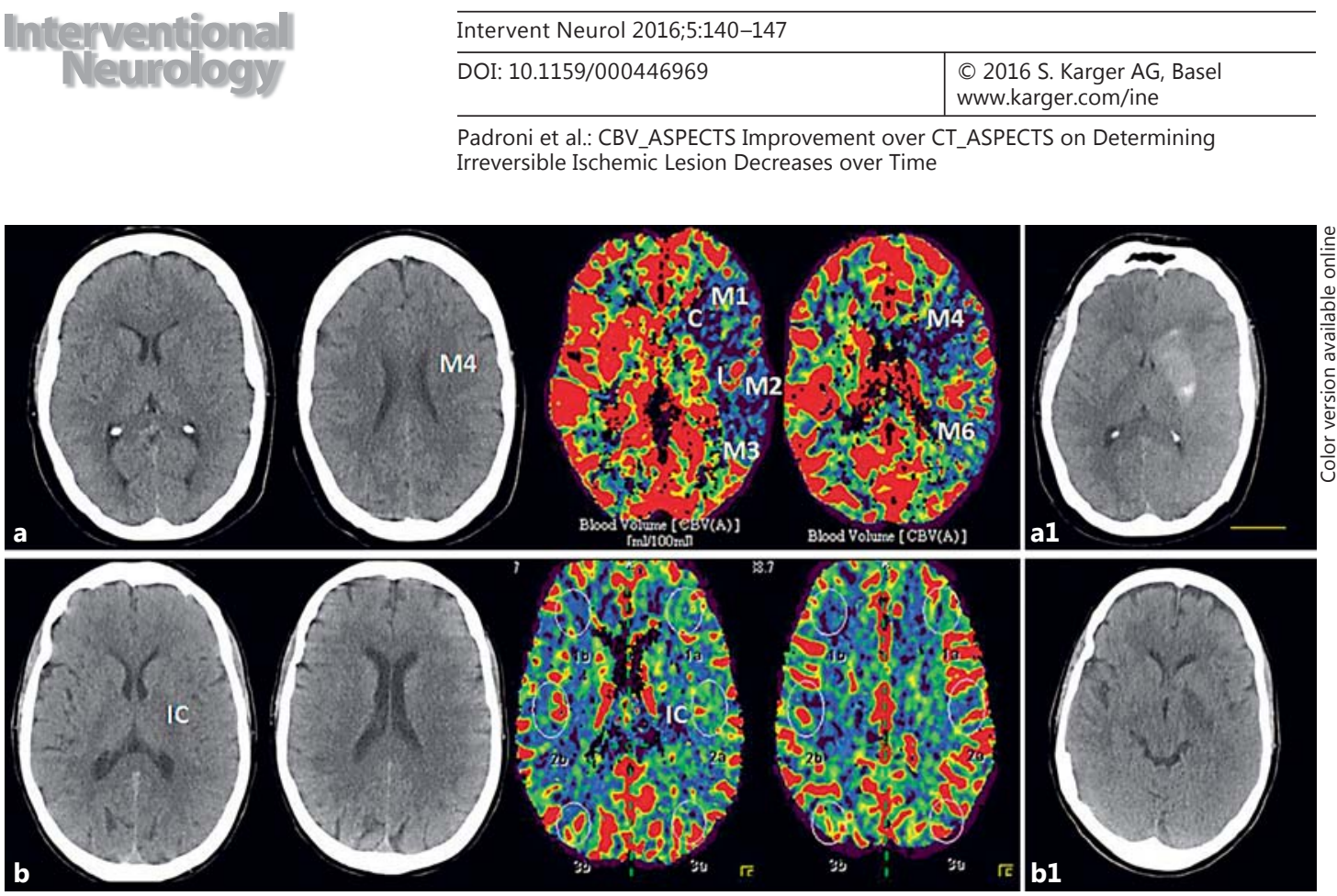

Fig. 1. CT_ASPECTS and CBV_ASPECTS comparison in 2 patients with ASPECTS disagreement (a) and ASPECTS agreement (b). a Patient with 110 min of global aphasia and right-side hemiparesis. Left M1-MCA occlusion treated with intravenous recombinant tissue plasminogen activator and endovascular reperfusion treatment, with complete recanalization after 215 min from symptom onset. b Patient with 200 min of mild right hemiparesis and motor aphasia. Distal left M1-MCA occlusion, with complete recanalization at $245 \mathrm{~min}$ from symptom onset after primary endovascular thrombectomy. a1, b1 24-hour CT scan showing final infarct lesion in both patients.

\section{Imaging Protocol}

Multimodal CT study was performed on Definition AS Siemens (Siemens, Erlangen, Germany). All patients underwent noncontrast CT to rule out hemorrhage and patients with large early parenchymal ischemic changes (ASPECTS <7). CTA was performed to determine the presence of large-vessel occlusion, and select patients for endovascular reperfusion treatments. Parameters for CTP were: collimator of $32 \times 1.5 \mathrm{~mm}, 80$ $\mathrm{kVp}$, and $200 \mathrm{mAs}$ with total coverage of $86 \mathrm{~mm}$. The plane of imaging was parallel to the floor of the anterior cranial fossa starting just above the orbits. Thirty cycles were obtained with a total scan time of $46 \mathrm{~s}$.

\section{Image Analysis}

ASPECTS was computed on nonenhanced CT by a radiologist in the emergency setting, and revised subsequently by two experienced neuroradiologists (P.C. and J.C). When there was a discrepancy, decision about the ASPECTS was taken by consensus.

All the images were transferred to a separate workstation for analysis using a DICOM viewer (Osirix 64-bit; Pixmeo, Geneva, Switzerland). CBV_ASPECTS was assessed later on on baseline CTP maps by a vascular neurologist (M.P.) blinded to CT_ASPECTS evaluation and clinical data. ASPECTS in noncontrast CT and CBV were compared. We defined as ASPECTS agreement a difference between CT_ASPECTS and CBV ASPECTS $\leq 1$. If the difference was higher than 1, it was considered as ASPECTS disagreement (fig. 1).

Final infarct volumes were measured on follow-up noncontrast CT scan at $24-48 \mathrm{~h}$ using the ABC/2 method [19].

\section{Statistical Analysis}

Descriptive and frequency statistical analysis were obtained using SPSS 17.0 software. Categorical variables are presented as absolute values and percentages, while the continuous variables are presented as median \pm SD if normally distributed, or median (interquartile ranges, IQR) if not normally distributed. Correlation between continuous variables was assessed by Spearman's correlation coefficient. A ROC curve analysis was used to calculate the best cutoff time point after which the ASPECTS difference becomes $\leq 1$. Statistical significance for intergroup differences was assessed by Pearson's $\chi^{2}$ or Fisher's exact test for categorical variables and by the Student $t$ test for continuous variables. Not normally distributed variables were evaluated by the Mann-Whitney $\mathrm{U}$ test. A p value $<0.05$ was considered significant for all tests. 
Table 1. Radiological and clinical data

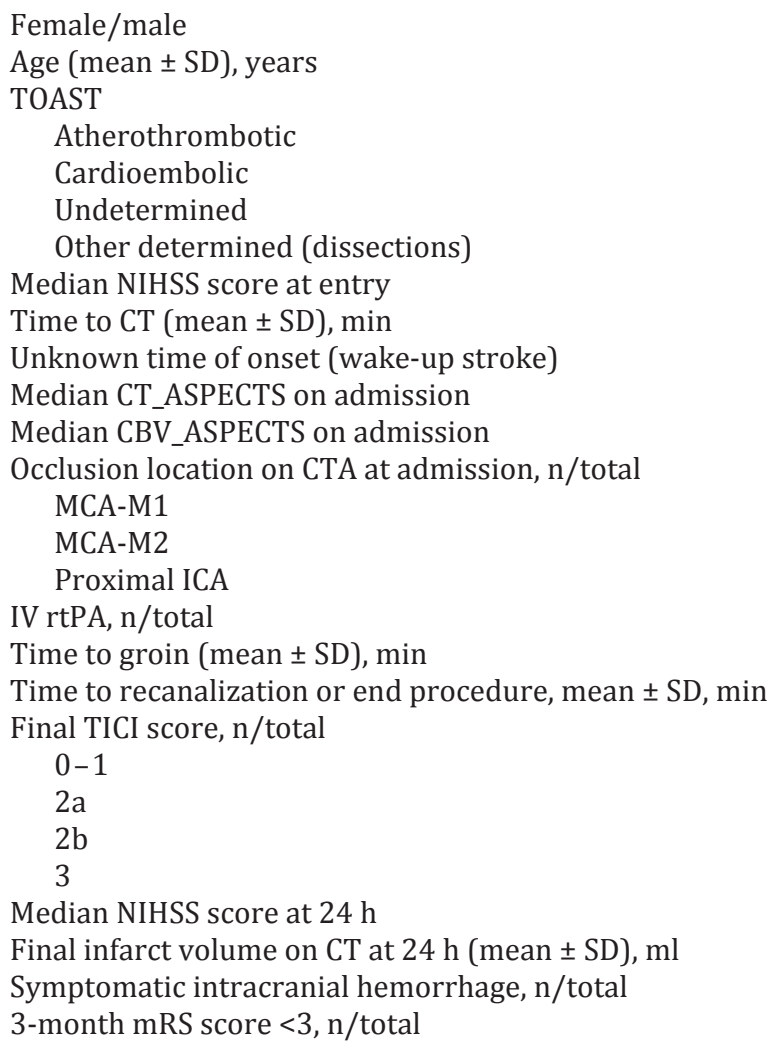

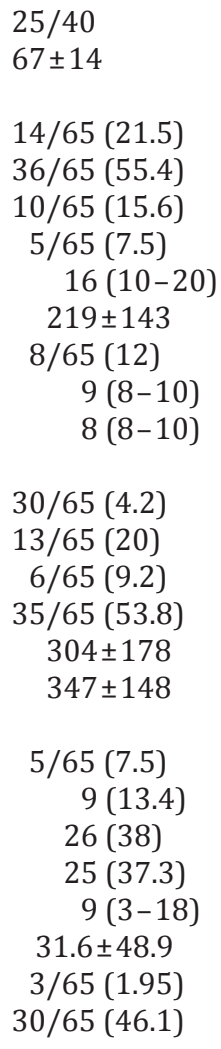

Figures in parentheses are percentages or IQR, unless indicated otherwise. TOAST = Trial of ORG 10172 in acute stroke treatment; IV rtPA = intravenous recombinant tissue plasminogen activator.

\section{Results}

Baseline clinical and radiological data are presented in table 1. Sixty-five patients $(25$ men), with a mean age of $67 \pm 14$ years (range 36-86) were studied. Median National Institutes of Health Stroke Scale (NIHSS) at onset was 16 (IQR 10-20).

Initial angiography identified 6 proximal ICA occlusions (9.2\%), 16 terminal ICA occlusions (24.6\%), 30 M1 occlusions (46.2\%) and 13 proximal M2 occlusions (20\%). All the patients underwent endovascular reperfusion treatment; 53.8\% (35 patients) after intravenous thrombolysis. Recanalization (defined as TICI score $2 \mathrm{~b}-3$ ) occurred in 51 patients (78.5\%). The median NIHSS score at $24 \mathrm{~h}$ was 9 (IQR 3-18). The mean infarct volume in the 24 - to 48 -hour control CT was $31.6 \pm 48.9 \mathrm{ml}$. Thirty patients $(46.1 \%)$ achieved a good functional outcome (mRS score $<3$ ) at 3 months (table 1 ).

Mean time from symptom onset to CT was $219 \pm 143$ min. On baseline CT, median CT_ ASPECTS was 9 (IQR 8-10), and in CTP, median CBV_ASPECTS was 8 (IQR 8-10). In 50 patients (76.9\%), the CT_ASPECTS and CBV_ASPECTS difference was $\leq 1$ (ASPECTS agreement).

We evaluated whether any clinical or radiological variable could be related to the presence of ASPECTS agreement. In the univariate analysis, ASPECTS disagreement was associated with lower CBV_ASPECTS and larger infarct volumes. Time to CT also differed significantly between patients with and without ASPECTS agreement (table 2). 
Padroni et al.: CBV_ASPECTS Improvement over CT_ASPECTS on Determining Irreversible Ischemic Lesion Decreases over Time

Table 2. Clinical and radiological comparison between patients with and without ASPECTS_agreement

\begin{tabular}{|c|c|c|c|}
\hline & $\mathrm{AD}$ & AA & $\mathrm{p}$ value \\
\hline Age (mean $\pm S D)$, years & $72 \pm 15$ & $65 \pm 13$ & 0.11 \\
\hline Female/male & $9 / 6$ & $31 / 19$ & 0.89 \\
\hline Hypertension & $10 / 15$ & $31 / 50$ & 0.74 \\
\hline Atrial fibrillation & $6 / 15$ & $13 / 50$ & 0.30 \\
\hline Diabetes mellitus & $3 / 15$ & $10 / 50$ & 1.00 \\
\hline CHD & $2 / 15$ & $6 / 50$ & 1.00 \\
\hline Dyslipidemia & $7 / 15$ & $18 / 50$ & 0.46 \\
\hline Statin therapy & $6 / 15$ & $17 / 50$ & 0.71 \\
\hline Previous stroke & $1 / 15$ & $11 / 50$ & 0.27 \\
\hline Glycemia (mean \pm SD), mg/dl & $122 \pm 34$ & $130 \pm 53$ & 0.61 \\
\hline Systolic blood pressure at onset (mean \pm SD), mm Hg & $150 \pm 29$ & $144 \pm 34$ & 0.58 \\
\hline Diastolic blood pressure at onset (mean \pm SD), mm Hg & $81 \pm 14$ & $78 \pm 17$ & 0.57 \\
\hline Time to CT (mean \pm SD), min & $150 \pm 141$ & $244 \pm 137$ & $0.02^{*}$ \\
\hline Time to $\mathrm{CT}<120 \mathrm{~min}$ & $10 / 15$ & $7 / 42$ & $<0.01^{*}$ \\
\hline Site of vessel occlusion & & & 0.25 \\
\hline MCA-M1 & $7 / 15$ & $23 / 50$ & \\
\hline MCA-M2 & $2 / 15$ & $11 / 50$ & \\
\hline Proximal ICA & $0 / 15$ & $6 / 50$ & \\
\hline Terminal ICA & $6 / 15$ & $10 / 50$ & \\
\hline Recanalization (TICI score $2 \mathrm{~b}-3$ ) & $12 / 15$ & $39 / 50$ & 1.00 \\
\hline Final infarct volume on $24 \mathrm{~h}$ CT (mean \pm SD), ml & $55 \pm 74$ & $24 \pm 35$ & $<0.01^{*}$ \\
\hline 3-month mRS score $<3$ & $6 / 15$ & $24 / 50$ & 0.77 \\
\hline
\end{tabular}

$\mathrm{AD}=$ ASPECTS disagreement; AA = ASPECTS agreement; $\mathrm{CHD}=$ coronary artery disease; TOAST = trial of Org 10172 in Acute Stroke Treatment. ${ }^{*} \mathrm{p}<0.05$ statistical significance for intergroup differences. It was assessed by Pearson's $\chi^{2}$ or Fisher's exact test for categorical variables and by Student $t$ test for continuous variables.

Fig. 2. Scatter plot showing ASPECTS difference according to time to CT.

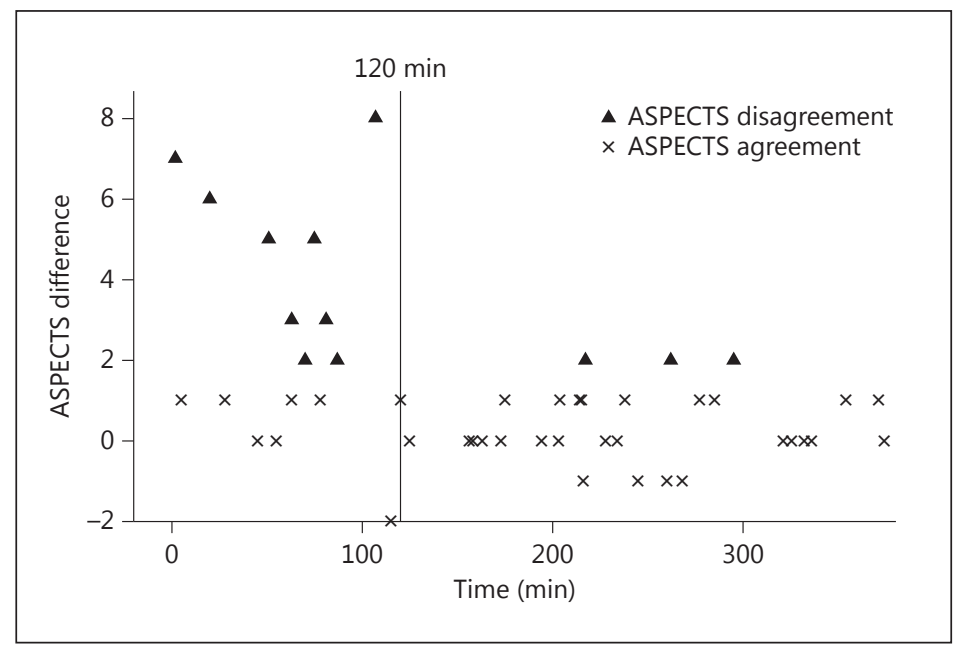

The ASPECTS difference was inversely correlated to the time from symptom onset to CT $(r=-0.36, p<0.01$ ). A ROC curve defined 120 min (sensibility: 0.83 , specificity: 0.67 ) as the best cutoff time point after which the ASPECTS difference becomes $\leq 1$. After $120 \mathrm{~min}$ (fig. 2), almost $90 \%$ of patients showed ASPECTS agreement ( 89.5 vs. $37.5 \%$ for $<120$ min; $p<0.01$ ). CBV_ASPECTS $(r=-0.33, p<0.01)$ but not CT_ASPECTS $(r<-0.20, p=0.08)$ correlated with 
the final infarct volume. However, if CT was done $>2 \mathrm{~h}$ after symptom onset, then CT_ASPECTS also correlated to the final infarct volume $(r=-0.39, \mathrm{p}=0.01)$.

Eight patients (12\%) presented with stroke of unknown time of onset because of wake-up stroke; all of them have ASPECTS agreement and were excluded for the time to CT analysis.

Median CT_ASPECTS was 10 (IQR 9-10) in patients who achieved a good functional outcome and 9 (IQR 8-10) in patients with poor functional outcome $(\mathrm{p}=0.12)$, while median CBV_ASPECTS was respectively 9 (IQR 8-10) and 8 (IQR 7-9) in these subgroups ( $p>0.05$ ). There was a trend towards higher CBV_ASPECTS in patients who achieved functional independency, but it did not reach statistical significance $(\mathrm{p}=0.09)$.

However, considering only patients who achieved recanalization, both CT and CBV ASPECTS correlate with 3-month mRS score ( $p=0.01$ and $p=0.04$, respectively).

\section{Discussion}

In this study, we explored the advantages of CBV_ASPECTS over CT_ASPECTS in the prediction of final infarct volume, as a surrogate marker of clinical outcomes [20,21]. Previous studies have shown a higher prognostic value of CTV_ASPECTS compared to CT_ASPECTS [14, $16,17,22]$ and this is confirmed by our data. In fact, CBV_ASPECTS but not CT_ASPECTS correlated to the final infarct volume. However, previous reports did not take into account the time from symptom onset to CT. Our study suggests that the predictive accuracy of CBV_ASPECTS is time-dependent. After $120 \mathrm{~min}$, CBV_ASPECTS did not provide additional information about ischemic core compared to CT_ASPECTS.

Early ischemic changes on baseline CT are related to the development of cytotoxic and subsequently ionic edema. The ability of the observers to detect these changes is influenced by the size of the infarction, the severity of ischemia and the time to CT [1, 23, 24]. It is known that CT reliability is lower in the ultra-early stroke presenters (within $90 \mathrm{~min}$ from symptom onset) [25]. As time goes by, the CT_ASPECTS is probably more accurate to detect these ischemic changes, and therefore, the ASPECTS difference decreases over time. On the other hand, CTP directly derives information on cerebral perfusion by analyzing the first passage through the cerebral vessels of an intravenous contrast bolus. The software generates the pixel-based color-coded parametric maps on the basis of the integration of the time density curves and deconvolution calculations [26]. Theoretically, the infarct core in CTP is the area with reduced cerebral blood flow and CBV (cerebral blood flow/CBV match) [27]. Using the ASPECTS methodology, we demonstrated that CTP was more reliable than plain CT at predicting final infarct volume in the first $2 \mathrm{~h}$ from symptom onset. In our series, all patients with unknown time of symptom onset (because of wake-up stroke) presented with ASPECTS agreement. We hypothesized that these patients were probably not early presenters.

However, our study showed that, when CT was done after $2 \mathrm{~h}$ from symptom onset, CT_ ASPECTS also correlated to the final infarct volume. In this time frame, CBV_ASPECTS was similar to CT_ASPECTS in almost $90 \%$ of the patients. Therefore, no additional information about ischemic core was provided by CBV in the majority of cases. Furthermore, ASPECTS disagreement was more frequent in patients with lower CBV_ASPECTS, and consequently, larger final infarct volumes.

The ischemic core is associated with the risk of hemorrhagic transformation and outcome after reperfusion therapies [18]. Theoretically, CTP adds information about the tissue at risk in the penumbral area. However, there are still uncertainties about which are the best CTP parameters to define core and penumbra $[28,29]$. Furthermore, recent evidences have shown the limited reliability of CTP in acute infarct volume measurements compared with multiparametric MRI [30]. 
The ESCAPE trial [31] selected patients for intra-arterial reperfusion therapies by using collateral assessment on CTA along with CT_ASPECTS. In concordance with our study, we believe that, after $2 \mathrm{~h}$ from symptom onset, CT_ASPECTS associated with CTA could be an adequate technique for selecting acute stroke patients for reperfusion therapies. This could represent a time, radiation and contrast-dose sparing imaging protocol for AIS patients who are not early presenters.

Our study presents several limitations; first of all its retrospective nature and the small sample size. Moreover, we performed an ASPECTS analysis of CBV maps visually, without using quantitative thresholds, and not a volumetric automated analysis similar to EXTEND-IA [10] or SWIFT PRIME [32] studies. Our findings should be confirmed in future larger studies, also using a volumetric analysis of the CBV maps, even better with standardized automated processing. Also, infarct volume was determined by the $\mathrm{ABC} / 2$ instead of volumetric measurements.

In conclusion, in acute stroke patients undergoing endovascular reperfusion therapies, baseline CBV_ASPECTS correlates with final infarct volume. However, when CT is performed beyond 120 min from symptom onset, CBV_ASPECTS does not add relevant information to CT_ASPECTS about the ischemic irreversible lesion.

\section{Statement of Ethics}

The study was approved by the local Ethics Committee. Informed consent was obtained from each patient or from his/her close relatives on admission. Details have been removed from the case descriptions to ensure anonymity.

\section{Disclosure Statement}

All the authors disclose no conflicts of interest related with this research. This research received no specific grant from any funding agency in the public, commercial or not-for-profit sectors.

\section{References}

1 Barber PA, Demchuk AM, Zhang J, Buchan AM: Validity and reliability of a computed tomography score in predicting outcome of hyperacute stroke before thrombolytic therapy. Lancet 2000;355:1670-1674.

2 Goyal M, Menon BK, Coutts SB, Hill MD, Demchuk AM; Penumbra Pivotal Stroke Trial Investigators, Calgary Stroke Program, and the Seaman MR Research Center: Effect of baseline CT scan appearance and time to recanalization on clinical outcomes in endovascular thrombectomy of acute ischemic strokes. Stroke 2011;42: 93-97.

3 Yoo AJ, Chaudhry ZA, Berkhemer OA, González RG, Goyal M, Demchuk AM, Menon BK, Mualem E, Ueda D, Buell H, Sit SP, Bose A; Penumbra Pivotal and Penumbra Imaging Collaborative Study (PICS) Investigators: Impact of pretreatment noncontrast CT Alberta Stroke Program Early CT Score on clinical outcome after intra-arterial stroke therapy. Stroke 2014;45:746-751.

4 Hill MD, Demchuk AM, Goyal M, Jovin TG, Foster LD, Tomsick TA, von Kummer R, Yeatts SD, Palesch YY, Broderick JP; IMS3 Investigators: Alberta Stroke Program early computed tomography score to select patients for endovascular treatment: Interventional Management of Stroke (IMS)-III Trial. Stroke 2014;45:444-449.

5 Dzialowski I, Hill MD, Coutts SB, Demchuk AM, Kent DM, Wunderlich O, von Kummer R: Extent of early ischemic changes on computed tomography (CT) before thrombolysis: prognostic value of the Alberta Stroke Program Early CT Score in ECASS II. Stroke 2006;37:973-978.

6 McTaggart RA, Jovin TG, Lansberg MG, Mlynash M, Jayaraman MV, Choudhri OA, Inoue M, Marks MP, Albers GW; DEFUSE 2 Investigators: Alberta stroke program early computed tomographic scoring performance in a series of patients undergoing computed tomography and MRI: reader agreement, modality agreement, and outcome prediction. Stroke 2015;46:407-412.

7 Gupta AC, Schaefer PW, Chaudhry ZA, Leslie-Mazwi TM, Chandra RV, González RG, Hirsch JA, Yoo AJ: Interobserver reliability of baseline noncontrast CT Alberta Stroke Program Early CT Score for intra-arterial stroke treatment selection. AJNR Am J Neuroradiol 2012;33:1046-1049.

8 Bivard A, Parsons M: ASPECTaSaurus (a dinosaur)? Int J Stroke 2012; 7:564. 
9 Puetz V, Dzialowski I, Hill MD, Demchuk AM: The Alberta Stroke Program Early CT Score in clinical practice: what have we learned? Int J Stroke 2009;4:354-364.

10 Campbell BC, Mitchell PJ, Kleinig TJ, Dewey HM, Churilov L, et al; EXTEND-IA Investigators: Endovascular therapy for ischemic stroke with perfusion-imaging selection. N Engl J Med 2015;12;372:1009-1018.

11 Kidwell CS, Jahan R, Gornbein J, Alger JR, Nenov V, Ajani Z, Feng L, Meyer BC, Olson S, Schwamm LH, Yoo AJ, Marshall RS, Meyers PM, Yavagal DR, Wintermark M, Guzy J, Starkman S, Saver JL; MR RESCUE Investigators: A trial of imaging selection and endovascular treatment for ischemic stroke. N Engl J Med 2013;7;368:914-923.

12 Wintermark M, Flanders AE, Velthuis B, Meuli R, van Leeuwen M, Goldsher D, Pineda C, Serena J, van der Schaaf I, Waaijer A, Anderson J, Nesbit G, Gabriely I, Medina V, Quiles A, Pohlman S, Quist M, Schnyder P, Bogousslavsky J, Dillon WP, Pedraza S: Perfusion-CT assessment of infarct core and penumbra: receiver operating characteristic curve analysis in 130 patients suspected of acute hemispheric stroke. Stroke 2006;37:979-985.

13 Murphy BD, Fox AJ, Lee DH, Sahlas DJ, Black SE, Hogan MJ, Coutts SB, Demchuk AM, Goyal M, Aviv RI, Symons S, Gulka IB, Beletsky V, Pelz D, Hachinski V, Chan R, Lee TY: Identification of penumbra and infarct in acute ischemic stroke using computed tomography perfusion-derived blood flow and blood volume measurements. Stroke 2006;37:1771-1777.

14 Parsons MW, Pepper EM, Chan V, Siddique S, Rajaratnam S, Bateman GA, Levi CR: Perfusion computed tomography: prediction of final infarct extent and stroke outcome. Ann Neurol 2005;58:672-679.

15 Copen WA, Morais LT, Wu O, Schwamm LH, Schaefer PW, González RG, Yoo AJ: In acute stroke, can CT perfusion-derived cerebral blood volume maps substitute for diffusion-weighted imaging in identifying the ischemic core? PLoS One 2015;10:e0133566.

16 Aviv RI, Mandelcorn J, Chakraborty S Gladstone D, Malham S, Tomlinson G, Fox AJ, Symons S: Alberta Stroke Program Early CT Scoring of CT perfusion in early stroke visualization and assessment. AJNR Am J Neuroradiol 2007;28:1975-1980.

17 Lin K, Rapalino O, Lee B, Do KG, Sussmann AR, Law M, Pramanik BK: Correlation of volumetric mismatch and mismatch of Alberta Stroke Program Early CT Scores on CT perfusion maps. Neuroradiology 2009;51:17-23.

18 Lum C, Ahmed ME, Patro S, Thornhill R, Hogan M, Iancu D, Lesiuk H, Dos Santos M, Dowlatshahi D; Ottawa Stroke Research Group (OSRG): Computed tomographic angiography and cerebral blood volume can predict final infarct volume and outcome after recanalization. Stroke 2014;45:2683-2688.

19 Sims JR, Gharai LR, Schaefer PW, Vangel M, Rosenthal ES, Lev MH, Schwamm LH: ABC/2 for rapid clinical estimate of infarct, perfusion, and mismatch volumes. Neurology 2009;72:2104-2110.

20 Padroni M, Bernardoni A, Tamborino C, et al: Cerebral blood volume ASPECTS is the best predictor of clinical outcome in acute ischemic stroke: a retrospective, combined semi-quantitative and quantitative assessment. PLos One 2016;11:e0147910.

21 Vagal AS, Sucharew H, Prabhakaran S, et al: Final infarct volume discriminates outcome in mild strokes. Neuroradiol J 2015;28:404-408.

22 Kloska SP, Dittrich R, Fischer T, Nabavi DG, Fischbach R, Seidensticker P, Osada N, Ringelstein EB, Heindel W: Perfusion CT in acute stroke: prediction of vessel recanalization and clinical outcome in intravenous thrombolytic therapy. Eur Radiol 2007;17:2491-2498.

23 Hirano T, Yonehara T, Inatomi Y, Hashimoto Y, Uchino M: Presence of early ischemic changes on computed tomography depends on severity and the duration of hypoperfusion: a single photon emission-computed tomographic study. Stroke 2005;36:2601-2608.

24 Demchuk AM, Coutts SB: Alberta Stroke Program Early CT Score in acute stroke triage. Neuroimaging Clin N Am 2005;15:409-419.

25 Bal S, Bhatia R, Menon BK, Shobha N, Puetz V, Dzialowski I, Modi J, Goyal M, Hill MD, Smith EE, Demchuk AM: Time dependence of reliability of noncontrast computed tomography in comparison to computed tomography angiography source image in acute ischemic stroke. Int J Stroke 2015;10:55-60.

26 Cianfoni A, Colosimo C, Basile M, Wintermark M, Bonomo L: Brain per-fusion CT: principles, technique and clinical applications. Radiol Med 2007;112:1225-1243.

27 Murphy BD, Fox AJ, Lee DH, Sahlas DJ, Black SE, Hogan MJ, Coutts SB, Dem-chuk AM, Goyal M, Aviv RI, Symons S, Gulka, IB, Beletsky V, Pelz D, Hachinski V, Chan R, Lee TY: Identification of penumbra and infarct in acute ischemic stroke using computed tomography perfusion-derived blood flow and blood volume measurements. Stroke 2006;37:1771-1777.

28 Campbell BC, Christensen S, Levi CR, Desmond PM, Donnan GA, Davis SM, Parsons MW: Cerebral blood flow is the optimal CT perfusion parameter for assessing infarct core. Stroke 2011;42:3435-3440.

29 Campbell BC, Christensen S, Levi CR, Desmond PM, Donnan GA, Davis SM, Parsons MW: Comparison of computed tomography perfusion and magnetic resonance imaging perfusion-diffusion mismatch in ischemic stroke. Stroke 2012;43:2648-2653.

30 Schaefer PW, Souza L, Kamalian S, Hirsch JA, Yoo AJ, Kamalian S, Gonzalez RG, Lev MH: Limited reliability of computed tomographic perfusion acute infarct volume measurements compared with diffusion-weighted imaging in anterior circulation stroke. Stroke 2015;46:419-424.

31 Goyal M, Demchuk AM, Menon BK, Eesa M, Rempel JL, Thornton J, et al; ES-CAPE Trial Investigators: Randomized assessment of rapid endovascular treatment of ischemic stroke. N Engl J Med 2015;372:1019-1030.

32 Saver JL, Goyal M, Bonafe A, Diener HC, et al; SWIFT PRIME Investigators: Stent-retriever thrombectomy after intravenous t-PA vs t-PA alone in stroke. N Engl J Med 2015;372:2285-2295. 